\title{
A 10-year survey of penetrating eye injuries in Gwent, 1976-85
}

\author{
W N WYKES \\ From the Department of Ophthalmology, University Hospital of Wales, Heath Park, Cardiff CF4 4XW
}

SUMMARY A retrospective survey was performed of all penetrating eye injuries in Gwent from 1976 to 1985 . It included 171 eyes. The results showed a gradual decline in all penetrating injuries and not just those due to road traffic accidents. An analysis of the cause and prognosis is given.

There have recently been several surveys of penetrating eye injuries in relation to the seat belt law which took effect in February 1983. ${ }^{1-3}$ These cover short periods before and after the introduction of the law and examine only road traffic accident cases (RTAs). Other surveys before 1976 have examined periods of several years and categorised the type of injury most prevalent in the population at the time. ${ }^{+7}$

The present survey was conducted to evaluate a clinical impression that apart from the fall in RTA injuries there has been a gradual decline in the number of penetrating injuries generally over the last few years and to examine any changing pattern of aetiology. The survey was conducted by examining the notes of all patients requiring surgery for penetrating injuries at St Woolos Hospital, Newport, Gwent. This is the sole unit for inpatient ophthalmic care in Gwent and has a current catchment population of 440000 .

\section{Materials and methods}

The survey included 171 eyes of 169 patients and covered the period 1 January 1976 to 31 December 1985. Patients were included in the study by examination of the operation books for this period and tracing the notes of all cases relating to ocular injury. Only those with penetrating injury were then included in this study. For each patient the age, sex, eye injured, aetiology, surgery, and final visual outcome were recorded, where available. Four sets of notes were not found (Table 1). These cases have not been included in any of the figures.

Correspondence to W N Wykes, FRCS.

\section{Results}

Age and sex. There was a marked preponderance of male patients throughout the age range. The overall numbers were 147 males and 22 females, a ratio of approximately 7:1. Fig. 1 shows the age distribution of the two sexes and that the male incidence is highest in the second to fourth decades. The female incidence is highest in the first two decades. In three male patients the age was unrecorded. The right eye was involved in 82 cases $(48 \%)$, and the left in 89 cases $(52 \%)$.

Aetiology. In all but two cases the aetiology was recorded. In children play and sport were the commonest cause of injury, accounting for $79.2 \%$ of all cases under 16 years, with home accidents $(14.6 \%)$ and assault $(6 \cdot 2 \%)$ accounting for the remainder (Fig. 2). Within the play and sport group are included 12 cases ( $25 \%$ of injuries under 16 years) due to darts, the commonest single factor in this group. Two of the cases over 16 years were also due to dart injuries. The incidence of dart injuries remained fairly constant throughout the survey and went against the general trend.

In adults industrial accidents were the commonest

Table 1 Notes not traceable

\begin{tabular}{lll}
\hline Year & Sex & Description \\
\hline 1977 & Male & Left enucleation and facial suturing \\
1982 & Male & Age 29, penetrating injury \\
1982 & Male & Penetrating injury, prolapsed iris \\
1982 & Male & Penetrating injury \\
\hline
\end{tabular}




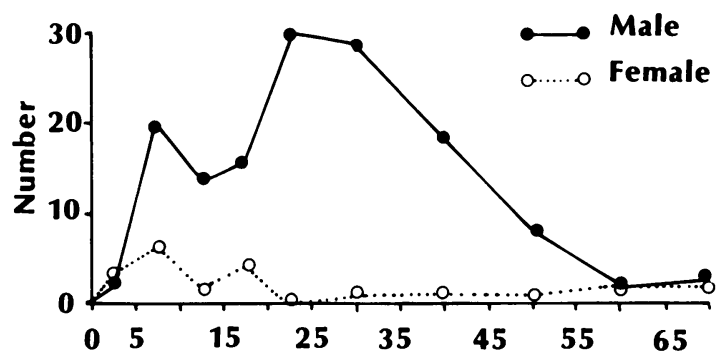

Age (years)

Fig. 1 Distribution of injured patients by age and sex.

cause, accounting for $53 \%$ of all cases over 16 years, while RTAs accounted for $21.3 \%$, mainly in the $16-24$ age group (68\% of RTAs) (Fig. 2). The remainder were due to home accidents $(12 \%)$, adult sport $(6 \%)$, and assault $(7 \cdot 7 \%)$. In three cases-one RTA and two industrial accidents - the age was unknown.

Temporal trends. One of the main aims of the survey was to see whether there was a gradual decline in injuries apart from RTAs. The seat belt law came into effect during the time period of the survey. Fig. 3 shows the total number of penetrating injuries for each year and the number minus those due to RTAs. This shows a steady decline in all injuries, particularly in 1984 and 1985, and shows the same trend when the RTA figures are removed. The RTA cases themselves show a steady decline before the seat belt law was introduced, and only two cases since, both in late 1983.

There was a steady decline in industrial accidents during the 10-year period (Fig. 4, Table 2). There has

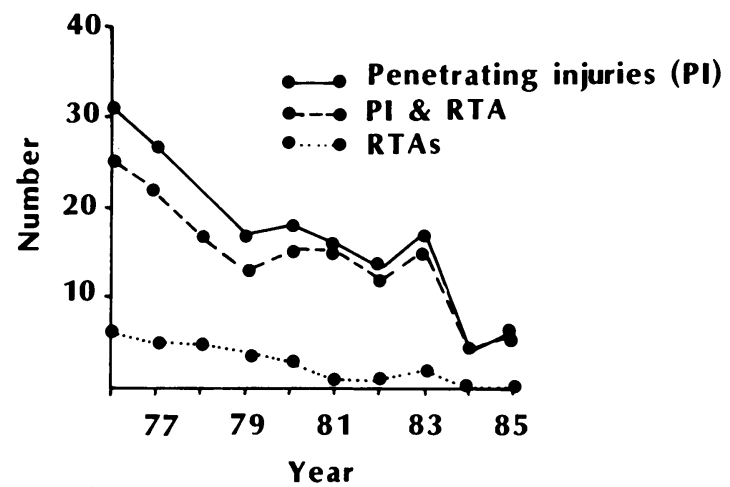

Fig. 3 Penetrating injuries and road traffic accidents.

also been some decline in play and sport injuries among children. However, the injuries due to darts (14 injuries, 12 in children under 16 years) show no trend. The injuries due to adult sport and assault show no overall trend (Table 2).

Visual prognosis. Examination of the final visual acuities revealed that nearly half the eyes $(43.3 \%)$ regained a visual acuity of $6 / 12$ or better (Table 3 ). However, almost a quarter $(24.5 \%)$ of the patients were completely blind-that is, had no perception of light $(6 \cdot 4 \%)$ - or had the eye enucleated $(18 \cdot 1 \%)$.

Primary enucleation was performed in three cases owing to extensive injuries. The indications for the remaining 28 enucleations are given in Table 4 . Of the causes of injury in the total of 31 enucleations play and sport (8) and industrial accident (8) were the commonest, with RTA (4), home accident (4), assault (4), and adult sport (3) accounting for the rest. Five of six eyes injured by airguns were enucleated, reflecting the severity of this type of

Fig. 2 Aetiology of ocularinjury.

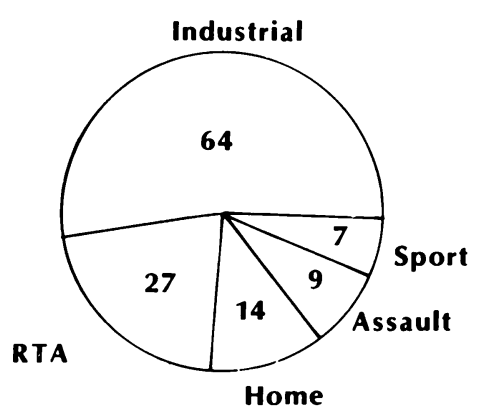

Total no. of patients $=121$

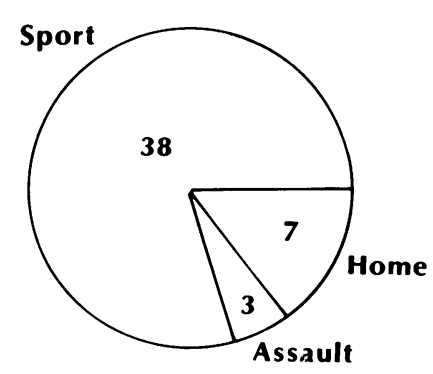

Total no. of patients - 48 


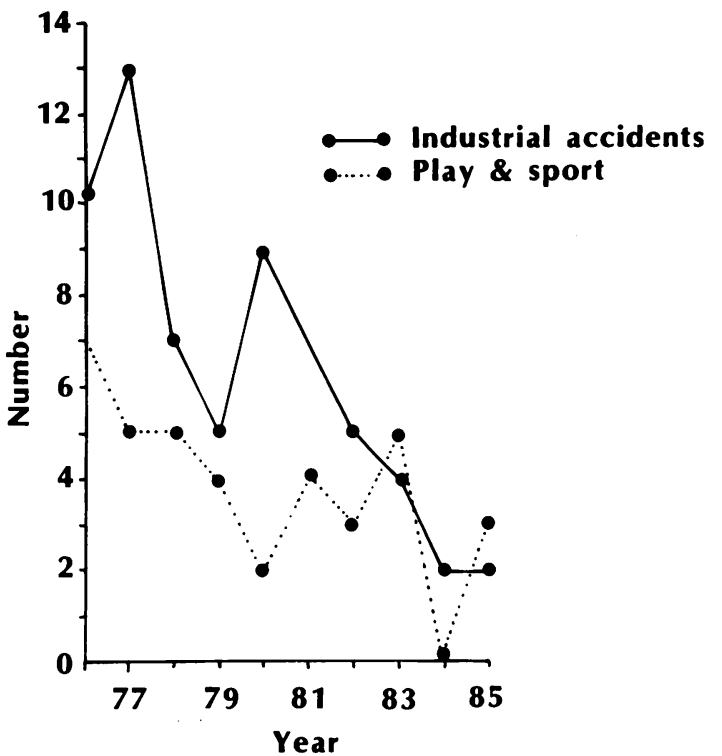

Fig. 4 Trends: industrial accidents: play and sport.

injury. Eleven of the eyes enucleated were in patients under 16 years old.

Sympathetic ophthalmitis occurred in four patients, one of whom, aged 6, presented with sympathetic ophthalmitis after a dart injury 10 days earlier. Of the other three, one had been involved in an RTA, one had been hit by a steel band at work, and one had been hit by a piece of wood.

Further operations. Seventy-six eyes (44.4\%) underwent further surgery, some having as many as four operations in all (Table 5). Cataract extraction $(29.6 \%)$ and enucleation $(25.9 \%)$ were the commonest operations. Other operations included trabeculectomy, resuturing of the conjunctiva after enucleation, and plastic surgery of the lids.

Intraocular foreign bodies. Thirty-seven cases $(21.6 \%)$ were of retained intraocular foreign bodies.
Hammering injuries accounted for over half $(56 \cdot 8 \%)$ of these (Table 6). A further fifth $(18.9 \%)$ were due to various causes involving small fragments of metal.

\section{Discussion}

The incidence of penetrating eye injuries due to RTAs has been shown to have fallen since the introduction of the seat belt law on 1 February 1983. ${ }^{1-3}$ Vernon and Yorston' reported a 58\% reduction in two six-month periods before and after the law, and Hall et al. ${ }^{2}$ found a $73 \%$ reduction comparing a two-year period before and after the law. Johnston and Armstrong ${ }^{3}$ reported a $60 \%$ reduction in Northern Ireland over the same period. However, the present survey indicates a steady decline in RTA injuries over the years preceding the law, with only two cases since. This may reflect a response to the publicity campaigns preceding the law and the controversy and resultant attention of the media surrounding the law itself.

A major purpose of the survey was to investigate the clinical impression that the number of penetrating injuries seen annually was steadily decreasing, apart from the reduction in RTAs. This is shown to be the case when the injuries due to RTAs are removed from the figures, in contrast to the figures produced by Johnston and Armstrong, ${ }^{3}$ who found that in 1981-3 there were 89 injuries from causes other than RTAs compared with 94 in 1983-5. The decline shown in this survey is mainly due to the decrease in injuries from industrial accidents, though there has been a slight decline in play and sport injuries among children.

In 1923 Garrow $^{8}$ reported that occupational injuries accounted for $70 \%$ of all eye injuries, but the Northern Ireland survey of $1967-76$ found that only $15.4 \%$ were industrial and that this rate was fairly constant over the 10 years. ${ }^{68}$ Johnston and Armstrong reported that $14 \%$ of perforating injuries without intraocular foreign body and $72 \%$ with

Table 2 Cause of injury by year

\begin{tabular}{|c|c|c|c|c|c|c|c|c|}
\hline & $\begin{array}{l}\text { Industrial } \\
\text { accident }\end{array}$ & $\begin{array}{l}\text { Play/sport } \\
\text { (children) }\end{array}$ & $R T A$ & Home accident & Assault & Adult sport & Unknown & Total \\
\hline 1976 & 10 & 7 & 6 & 3 & 2 & 3 & - & 31 \\
\hline 1977 & 13 & 5 & 5 & 1 & 3 & - & - & 27 \\
\hline 1978 & 7 & 5 & 5 & 4 & - & 1 & - & 22 \\
\hline 1979 & 5 & 4 & 4 & 2 & 1 & 1 & - & 17 \\
\hline 1980 & 9 & 2 & 3 & 4 & - & - & - & 18 \\
\hline 1981 & 7 & 4 & 1 & 3 & - & 1 & - & 16 \\
\hline 1982 & 5 & 3 & 1 & - & 2 & - & 2 & 13 \\
\hline 1983 & 4 & 5 & 2 & 4 & 2 & - & - & 17 \\
\hline 1984 & 2 & - & - & - & 1 & 1 & - & 4 \\
\hline 1985 & 2 & 3 & - & - & 1 & - & - & 6 \\
\hline Total & 64 & 38 & 27 & 21 & 12 & 7 & 2 & 171 \\
\hline$\%$ & $37 \cdot 4$ & $22 \cdot 2$ & $15 \cdot 8$ & $12 \cdot 3$ & $7 \cdot 0$ & $4 \cdot 1$ & $1 \cdot 2$ & 100 \\
\hline
\end{tabular}


Table 3 Final visual acuity

\begin{tabular}{lrr}
\hline Acuity & No. & $\%$ \\
\hline 6/6 or better & 36 & $21 \cdot 1$ \\
$6 / 7 \cdot 5-6 / 12$ & 38 & $22 \cdot 2$ \\
$6 / 18-6 / 36$ & 16 & $9 \cdot 4$ \\
6/60 or worse & 22 & $12 \cdot 9$ \\
Noperception of light & 11 & $6 \cdot 4$ \\
Enucleation & 31 & $18 \cdot 1$ \\
Unrecorded & 17 & $9 \cdot 9$ \\
Total & 171 & 100 \\
\hline
\end{tabular}

Table 4 Indication for enucleation

\begin{tabular}{lrr}
\hline & No. & $\%$ \\
\hline Eye damaged beyond repair & 3 & $9 \cdot 7$ \\
Blind painful eye & 23 & $74 \cdot 2$ \\
Phthisis & 3 & $9 \cdot 7$ \\
Endophthalmitis & 2 & $6 \cdot 4$ \\
Total & 31 & 100 \\
\hline
\end{tabular}

Table 5 Furtheroperations

\begin{tabular}{lccccc}
\hline Operation & 2nd op & 3rdop. & 4thop. & Total & $\%$ \\
\hline Enucleation & 23 & 4 & 1 & 28 & $25 \cdot 9$ \\
Cataract extraction & 29 & 3 & - & 32 & $29 \cdot 6$ \\
Capsulotomy & 6 & 8 & 2 & 16 & $14 \cdot 8$ \\
Retinal detachment & 5 & 3 & 1 & 9 & $8 \cdot 4$ \\
Squint & 2 & 5 & - & 7 & $6 \cdot 5$ \\
Other & 11 & 3 & 2 & 16 & $14 \cdot 8$ \\
Total & 76 & 26 & 6 & 108 & 100 \\
\hline
\end{tabular}

Table 6 Intraocular foreign bodies

\begin{tabular}{lrr}
\hline Cause & No. & $\%$ \\
\hline Hammer and chisel & 17 & $46 \cdot 0$ \\
Hammer and nail & 4 & $10 \cdot 8$ \\
Metal from unknown cause & 7 & $18 \cdot 9$ \\
Airgun pellet & 2 & $5 \cdot 4$ \\
Shotgun pellet & 2 & $5 \cdot 4$ \\
Plaster & 2 & $5 \cdot 4$ \\
Other & 3 & $8 \cdot 1$ \\
Total & 37 & 100 \\
\hline
\end{tabular}

were industrial. ${ }^{3}$ In this survey of penetrating injuries $37.4 \%$ were industrial, but this reflects a reduction in other causes rather than an increase in industrial accidents, and the decline during the 10 years is heartening.

The male/female ratio of $6 \cdot 68: 1$ is in keeping with other surveys. Canavan et al. ${ }^{6}$ found a ratio of 5.25:1, and Blomdahl and Norrell ${ }^{9}$ found a ratio of nearly 6:1.

In those patients whose visual outcome was known $43.3 \%$ had a final visual acuity of $6 / 12$ or better, compared with a range of $21-68 \%$ in other studies; $24.5 \%$ were rendered completely blind (including those with enucleated eyes) compared with $12 \cdot 2-$

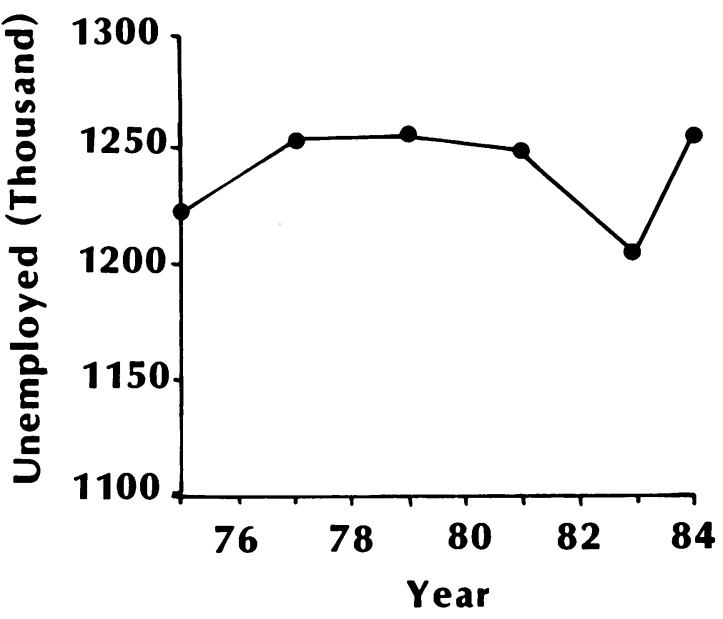

Fig. 5 Unemployment in Wales.

$31 \%$, and the enucleation rate was $18 \cdot 1 \%$ compared with $9 \cdot 2-28 \% .^{45711}$ Of the enucleated eyes a third $(35.5 \%)$ related to children, five being due to air rifle injuries and two to dart injuries. Overall, darts accounted for 14 injuries, 12 of them in patients under 16 years, and this was the commonest single cause of injury in children.

Four cases of sympathetic ophthalmitis occurred of which one already had sympathetic involvement on presentation. Johnston ${ }^{4}$ found two cases in his survey of 376 cases despite the excision rate of $28 \%$. Therefore, although the incidence is low, sympathetic ophthalmitis is still a real danger which must be borne in mind when treating penetrating injuries.

The possibility that the decline in industrial injuries was due to a fall in employment was considered. Specific figures for unemployment in Gwent were not available over the period, but the unemployment figures for Wales do not show any correlation with the decline in injuries (Fig. 5)." However, there may have been a shift in the type of employment away from heavy engineering.

\section{CONCLUSION}

This survey has shown a gradual decline in all penetrating injuries over the 10 -year period from 1976 to 1985 , not simply a reduction in RTA cases. The major causes throughout are industrial accidents in adults and play and sport in children. Both groups show a decline in incidence over the period which is particularly marked in the case of industrial injuries. Among the children dart injuries are the single most common cause and they do not show any decline in frequency, which is most distressing. They are clearly not subject to the same factors involved in the general decline, and perhaps specifically drawing attention to this problem in the media would help. 


\section{References}

1 Vernon SA, Yorston DB. Incidence of ocular injuries from road traffic accidents after introduction of seat belt legislation. $J R$ Soc Med 1984; 77: 198-200.

2 Hall NF, Denning AM, Elkington AR, Cooper PJ. The eye and the seatbelt in Wessex. Br J Ophthalmol 1985; 69: 317-9.

3 Johnston PB, Armstrong MFJ. Eye injuries in Northern Ireland two years after seat belt legislation. Br J Ophthalmol 1986; 70: $460-2$.

4 Johnston S. Perforating eye injuries: a five year survey. Trans Ophthalmol Soc UK 1971; 91: 895-921.

5 Horan EC. Perforating injuries in Cork. A review. Trans Ophthalmol Soc UK 1979; 99: 511-4.

6 Canavan YM, O'Flaherty MJ, Archer DB, Elwood JH. A 10- year survey of eye injuries in Northern Ireland, 1967-76. Br J Ophthalmol 1980; 64: 618-25.

7 Eagling EM. Perforating injuries of the eye. BrJ Ophthalmol $1980 ; 64: 618-25$

8 Garrow A. A statistical enquiry into 1000 cases of eye injuries. BrJ Ophthalmol 1923; 7: 65-80.

9 Blomdahl S, Norell S. Perforating eye injury in the Stockholm population: an epidemiological study. Acta Ophthalmol (Kbh) 1984; 62/3: 378-90.

10 Adhikary HP, Taylor P, Fitzmaurice DJ. Prognosis of perforating cye injury. BrJ Ophthalmol 1976; 60: 737-9.

11 Department of Employment. Employment Gazette 1986: February: 75. London: HMSO

Accepted for publication 4 June 1987. 